\title{
Microbial community analysis in biocathode microbial fuel cells packed with different materials
}

\author{
Yanmei Sun, Jincheng Wei, Peng Liang and Xia Huang*
}

\begin{abstract}
Biocathode MFCs using microorganisms as catalysts have important advantages in lowering cost and improving sustainability. Electrode materials and microbial synergy determines biocathode MFCs performance. In this study, four materials, granular activated carbon (GAC), granular semicoke (GS), granular graphite (GG) and carbon felt cube (CFC) were used as packed cathodic materials. The microbial composition on each material and its correlation with the electricity generation performance of MFCs were investigated. Results showed that different biocathode materials had an important effect on the type of microbial species in biocathode MFCs. The microbes belonging to Bacteroidetes and Proteobacteria were the dominant phyla in the four materials packed biocathode MFCs. Comamonas of Betaproteobacteria might play significant roles in electron transfer process of GAC, GS and CFC packed biocathode MFCs, while in GG packed MFC Acidovorax may be correlated with power generation. The biocathode materials also had influence on the microbial diversity and evenness, but the differences in them were not positively related to the power production.
\end{abstract}

Keywords: Biocathode microbial fuel cell, Cathodic materials, Electricity generation, Microbial community

\section{Introduction}

Microbial fuel cells (MFCs) utilize microorganisms as catalysts, which can promote biodegradation of organic matters and simultaneously produce an electrical current (Bond et al. 2002). In the past few years, researchers generally use chemical cathode MFC to remove the organic carbon in wastewater, but the cost of chemical cathode is high and it is easily lead to pollution. Currently, biocathode MFCs using microorganisms instead of common Pt as catalysts have important advantages in lowering cost, expanding function and improving sustainability. Therefore, biocathode MFCs as a new economical and environmentally friendly wastewater treatment technology has drawn more and more attentions (Huang et al. 2011). Although biocathode MFCs have many advantages, the current studies are still at laboratory level. The main challenge for their large-scale application is low power generation capability. Microorganisms are the core of biocathode MFCs. In the anode,

\footnotetext{
* Correspondence: xhuang@tsinghua.edu.cn

State Key Joint Laboratory of Environment Simulation and Pollution Control, School of Environment, Tsinghua University, Beijing 100084, P. R. China
}

microorganisms attaching on the electrode material and forming biofilm play an essential role in MFC generating electricity (Rabaey and Rozendal 2010), and in the cathode, the microbial catalytic efficiency plays a key role to improve the cathode potential and power output (Osman et al. 2010). Therefore, better understanding of the ecology of the microbial communities in the different reactors will be helpful to improve MFCs power production.

At present, the anodic microbes get more attention, including the electricity-producing bacteria species (Holmes et al. 2004Xia et al. 2010), anodic microbial community composition (Cárcer et al. 2011Jung and Regan 2010Kim et al. 2011Zhang et al. 2011), the mechanism of extracellular electron transfer (CarmonaMartinez et al. 2011Strycharz et al. 2011) and so on. In contrast, the researches on the microbes of biocathode MFCs are very limited, and mainly focused on the role of pure bacteria in biocathode MFCs. For instance, Carbajosa et al. (2010) found that an acidophilic Acidithiobacillus ferrooxidans could promote oxygen reduction in biocathode MFCs. Mao et al. (2010) reported that the 
power generation from a biocathode MFC was biocatalyzed by ferro/manganese-oxidizing bacteria. Recently, a research analyzed the microbial community and electron transfer, when nitrate was used as electron acceptor (Chen et al. 2010). However, electrode materials and microbial synergy determines biocathode MFCs performance. Different electrode materials have certain differences in conductivity, surface area and porosity. These differences may affect the cathode microbial adhesion and growth. However, the influence of different biocathode materials on the microbial composition is still unknown.

In our previous study (Wei et al. 2011), two types of relative cheaper electrode materials, granular semicoke (GS) and granular activated carbon (GAC), as biocathode packed materials, and the material characteristic, electrochemical performance and price-performance ratio were compared with carbon felt cube (CFC) and granular graphite (GG). Results indicated that MFCs with GS and GAC outperformed MFCs with GG and CFC biocathode. But the dominate microorganisms in different biocathode materials were not analyzed and the interaction mechanism between microbes and biocathode materials was unclear.

The objective of this study is to analyze the microbial community composition attaching on the four biocathode materials, illustrate the predominate microbes on each biocathode materials and analyze the relationship between microorganisms and power generation in biocathode MFCs.

\section{Materials and methods MFC construction and operation}

Four double-chambered flat plate MFCs with same size were built. Each MFC had two compartments with a total volume of $100 \mathrm{~mL}\left(2 \mathrm{~cm}\right.$ thickness, $50 \mathrm{~cm}^{2}$ cross section), which were separated by an Ultrex cation exchange membrane (CMI-7000, Membranes International, USA). The titanium mesh was placed next to the cation exchange membrane, which was used to gather electrons flowing in each chamber. The titanium sheet was served as a lead to connect both electrodes and external resistance. Four biocathode materials (CFC, GG, GAC and GS) were filled in separate cathodic compartments, and anodic compartments of all four MFCs were filled with the same CFC used in cathode. The anodic and cathodic compartments were inoculated with microbial consortiums previously enriched in biocathode MFCs that had been operated in fed-batch mode for over three months. Two saturated calomel electrodes (SCE, $0.242 \mathrm{~V}$ vs. standard hydrogen electrode (SHE), Leici, China) were fitted through the rubber stopper of anodic and cathodic compartments respectively to be used as reference electrodes. The medium in the anodic compartment consisted of $1.64 \mathrm{~g} /$ $\mathrm{L} \mathrm{CH}_{3} \mathrm{COONa}, 1.5 \mathrm{~g} / \mathrm{L} \mathrm{NH}_{4} \mathrm{Cl}, 3.4 \mathrm{~g} / \mathrm{L} \mathrm{KH}_{2} \mathrm{PO}_{4}, 4.4 \mathrm{~g} / \mathrm{L}$ $\mathrm{KH}_{2} \mathrm{PO}_{4}, 0.1 \mathrm{~g} / \mathrm{L} \mathrm{CaCl} \cdot 2 \mathrm{H}_{2} \mathrm{O}$ and $0.1 \mathrm{~g} / \mathrm{L} \mathrm{MgCl}_{2} \cdot 6 \mathrm{H}_{2} \mathrm{O}$. The cathode was fed with a similar medium with the anode, except that $1.64 \mathrm{~g} / \mathrm{L} \mathrm{CH}_{3} \mathrm{COONa}$ was replaced with $1.90 \mathrm{~g} / \mathrm{L} \mathrm{NaHCO}_{3}$. The nutrient solution was pumped to anodic and cathodic compartments separately at a rate of $20 \mathrm{~mL} / \mathrm{min}$ using a peristaltic pumps (BT100-1 L, Baoding Longer Precision Pump Co., Ltd., China). To provide abundant oxygen for the cathodic reaction, air was continuously sparged into a conical flask which was connected with the vessel of cathode recirculation. MFCs were operated in fed-batch mode, and the anolyte and catholyte were replaced every 3 days. All reactors were operated at ambient temperature $\left(28 \pm 3^{\circ} \mathrm{C}\right)$ with a $1000 \Omega$ resistor connected unless otherwise specified.

\section{Material characteristics and electrochemical analysis}

The specific surface area of four cathodic materials was measured with a micropore surface area and pore size analyzer (Autosorb-1 MP, Quantachrome Instrumants, USA).

The voltage across an external resistor was measured at a time interval of $20 \mathrm{~min}$ using a data acquisition system (DAQ2213, ADLINK, Beijing, China). The power densities were calculated from $P=I E / V$, where $I$ is the current $(=E / R), E$ the measured voltage, $R$ is the external resistance, $P$ the power density, and $V$ the net liquid volume of the anodic compartment (Logan et al., 2006).

\section{Bacterial community analysis}

Biofilms attaching on the biocathode materials in MFCs were sampled at the end of the experiment, and rinsed with sterile distilled water to remove loosely attached bacteria. The cathodic biofilm DNA was extracted using a PowerSoil DNA isolation kit (MO BIO Laboratories) and then the bacteria 16S rRNA was amplified. Bacterial universal primers 27f (5'-AGA GTT TGA TCM TGG CTC AG-3') and 1495r (5'-CTA CGG CTA CCT TGT TAC GA-3') (Barberio et al. 2001) were used, and the PCR conditions were carried out in accordance with the reference (Di Cello et al. 1997). The products of triplicate PCR amplifications from each sample were joined and purified with Wizard ${ }^{\mathbb{B}}$ SV Gel and PCR Clean-Up System (Promega). The purified product was ligated into the $\mathrm{pGEM}^{\circledR}-\mathrm{T}$ Easy vector (Promega) and the resulting plasmids were used to transform competent E. coli JM109 cells. Transformants were screened using bluewhite selection on Luria agar containing

X-gal/IPTG and $100 \mathrm{mg} / \mathrm{mL}$ ampicillin. White colonies were selected, transferred to fresh ampicillin-supplemented plates and incubated overnight at $37^{\circ} \mathrm{C}$. Plasmids were extracted using EZ Spin Column Plasmid 
Mini-Preps Kit (Shanghai Sangon). Positive clones were sequenced with primers T7/SP6 by a DNA sequencer (ABI 3730). Clone sequences were identified by comparison to the Genbank nucleotide database using BLAST via the National Center for Biotechnology Information (NCBI) (http://www.ncbi.nlm.nih.gov/BLAST/). Mega 4.0.2 (Tamura et al. 2007) was used to align these sequences, and the neighbor-joining method was employed to generate a phylogenetic tree with a bootstrap test (1000 replicates) of phylogeny. Sequences obtained in this study have been deposited in GenBank with accession numbers of JN541127-JN541192 and JN565978. Sequences with greater than 97\% identity are typically assigned to the same species (Schloss and Handelsman 2005). Rarefaction curves were used to determine if a sufficient number of clones from each of the libraries had been sequenced (http://strata.uga.edu/software/Software.html). The sampling coverage value, diversity (Shannon-Wiener index) and evenness were calculated as previously described (Gremion et al. 2003).

\section{Results}

\section{MFC performance}

The maximum power densities of four different cathodic materials packed MFCs are shown in Additional file 1: Table S1. Results indicated that as cathodic material, the power density of GAC was the highest $\left(24.3 \mathrm{~W} / \mathrm{m}^{3}\right)$, followed by GS $\left(20.1 \mathrm{~W} / \mathrm{m}^{3}\right)$, CFC $\left(17.1 \mathrm{~W} / \mathrm{m}^{3}\right)$, and GG $\left(14.4 \mathrm{~W} / \mathrm{m}^{3}\right)$.

\section{Diversity estimation and clone library comparison}

Four $16 \mathrm{~S}$ rRNA gene libraries were constructed to analyze the effect of biocathode materials on microbial composition. About 60 positive clones were sequenced in each library. Rarefaction curves (Additional file 1: Figure S1) showed that the number of clones was high enough to cover almost all the OTUs (operational taxonomic units) in samples. The coverage value for each clone library was more than $80 \%$, which also indicated that the four clone libraries could reflect the microbial community composition in samples. There were 22, 21, 16 and 13 genotypes in GS, CFC, GG and GAC clone libraries, respectively (Table 1). In GS packed MFCs, microbial diversity was the highest, the Shannon-Weaver index was 2.70, followed by the GG and CFC packed
MFCs, and GAC packed MFC had the lowest microbial diversity, about 1.83. Species evenness distributed on each electrode material had the similar trend to diversity. In GS packed MFC, the distribution of species was relatively average compared with other packed materials (Table 1).

\section{Microbial community distribution}

The microbial community distribution on each biocathode material was compared and four phylogenetic trees were constructed to characterize the relationship between those clone sequences. Results showed that microorganisms in the GAC packed MFC could be assigned into four groups, consisting of Alphaproteobacteria, Betaproteobacteria, Bacteroidetes and Acidobacteria (Figure 1). In the GS packed MFC, the cathodic biofilm was dominated by Alphaproteobacteria, Betaproteobacteria, Gammaproteobacteria, Bacteroidetes and Verrucomicrobia (Figure 2). The microorganisms belonged to Alphaproteobacteria, Betaproteobacteria, Gammaproteobacteria. Bacteroidetes also existed in GG packed MFC. In addition to the bacterial phyla, Acidobacteria and Actinobacteria were also found in GG (Figure 3). Alphaproteobacteria Betaproteobacteria, Bacteroidetes and Verrucomicrobia were dominant phyla attached to CFC (Figure 4).

The relative abundance of each phylum on the four biocathode materials was analyzed and compared in Figure 5. Results suggested that Bacteroidetes and Betaproteobacteria were the most abundant in the biofilms sampled from four cathode materials (Figure 5). The two phyla accounted for more than $80 \%$ of the total population in each biocathode material. The relative abundances of Alphaproteobacteria and Gammaproteobacteria were higher in GS compared with other materials. Actinobacteria was only existed in the GG cathodic material.

To visualize specific species and relative abundance within each electrode material, column chart was performed to illustrate the microbial community composition, as shown in Figure 6. The dominate genus and relative abundance varied significantly among the four cathodic materials. In the GAC packed MFC, clones showed a great similarity to Comamonas, and the amount of Comamonas accounted for $41 \%$ of the population. In addition, Acidovorax attaching on the GAC packed MFC,

Table 1 Bacterial 16S rRNA clone libraries from different cathode material packed in MFCs

\begin{tabular}{lllllll}
\hline Material & Transformant number & Positive clones number & OTUs & Coverage (\%) & Shannon index $(\boldsymbol{H})$ & Evenness (E) \\
\hline GS & 70 & 63 & 22 & 84.62 & 2.70 & 0.63 \\
\hline GG & 68 & 67 & 21 & 80.60 & 2.49 & 0.53 \\
\hline CFC & 59 & 55 & 16 & 83.64 & 2.25 & 0.51 \\
\hline GAC & 72 & 68 & 13 & 89.71 & 1.83 & 0.41 \\
\hline
\end{tabular}

OTUs: operational taxonomic units 

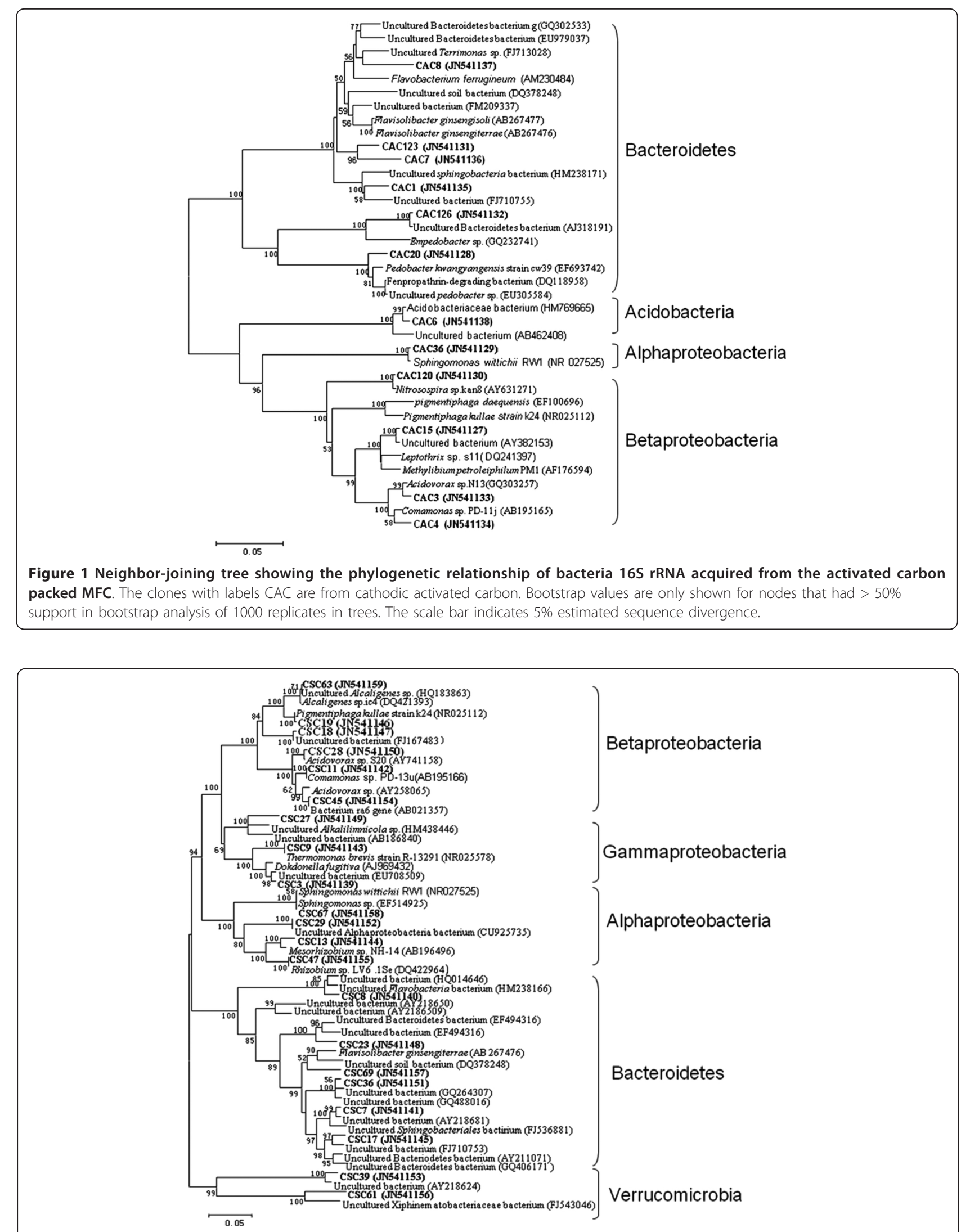

Figure 2 Neighbor-joining tree showing the phylogenetic relationship of bacteria 16S rRNA acquired from the semicoke packed MFC. The clones with labels CSC are from cathodic semicoke. Bootstrap values are only shown for nodes that had $>50 \%$ support in bootstrap analysis of 1000 replicates in trees. The scale bar indicates 5\% estimated sequence divergence. 


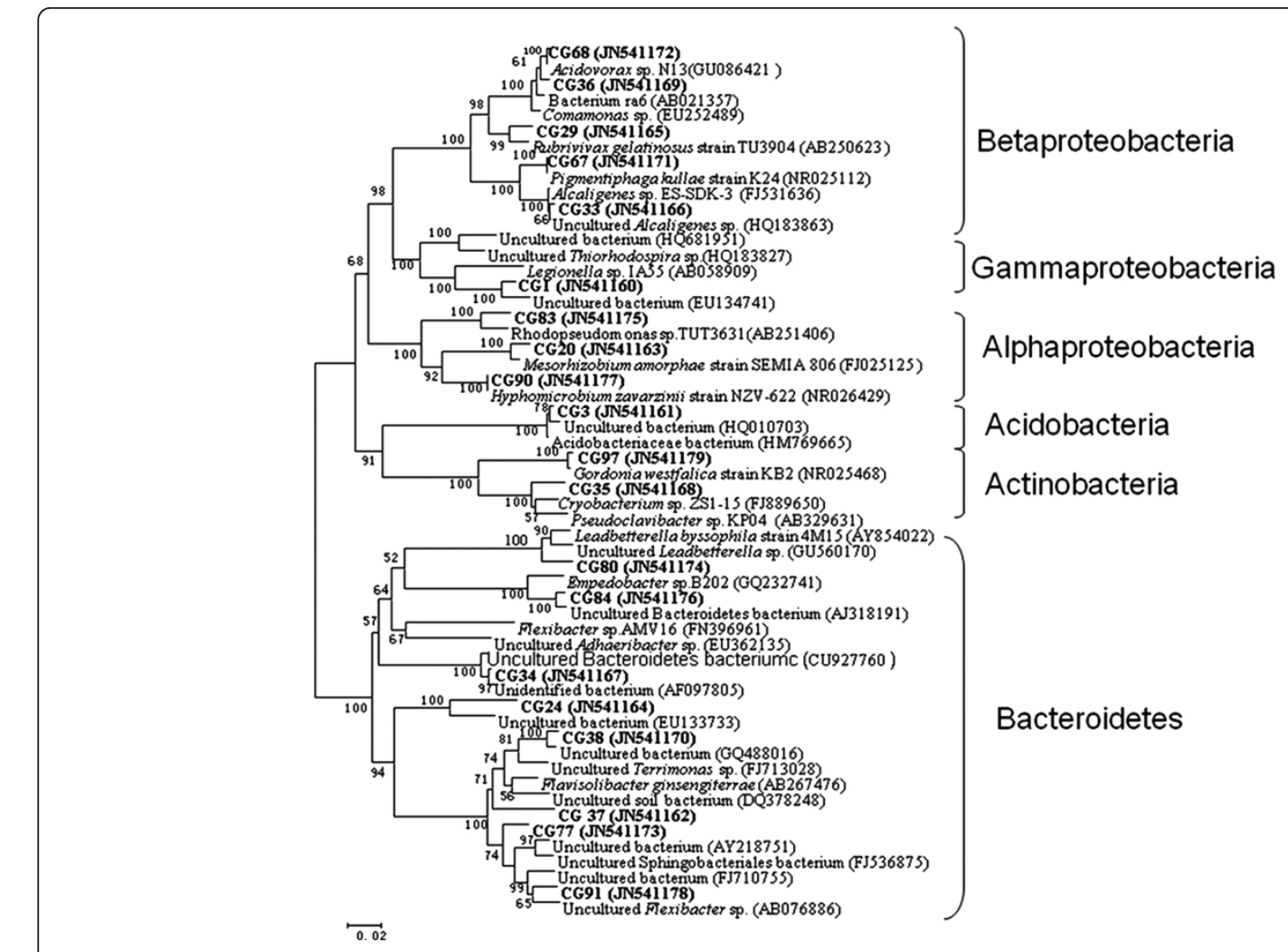

Figure 3 Neighbor-joining tree showing the phylogenetic relationship of bacteria 16S rRNA acquired from graphite packed MFC. The clones with labels CG are from cathodic graphite. Bootstrap values are only shown for nodes that had > 50\% support in bootstrap analysis of 1000 replicates in trees. The scale bar indicates $2 \%$ estimated sequence divergence.

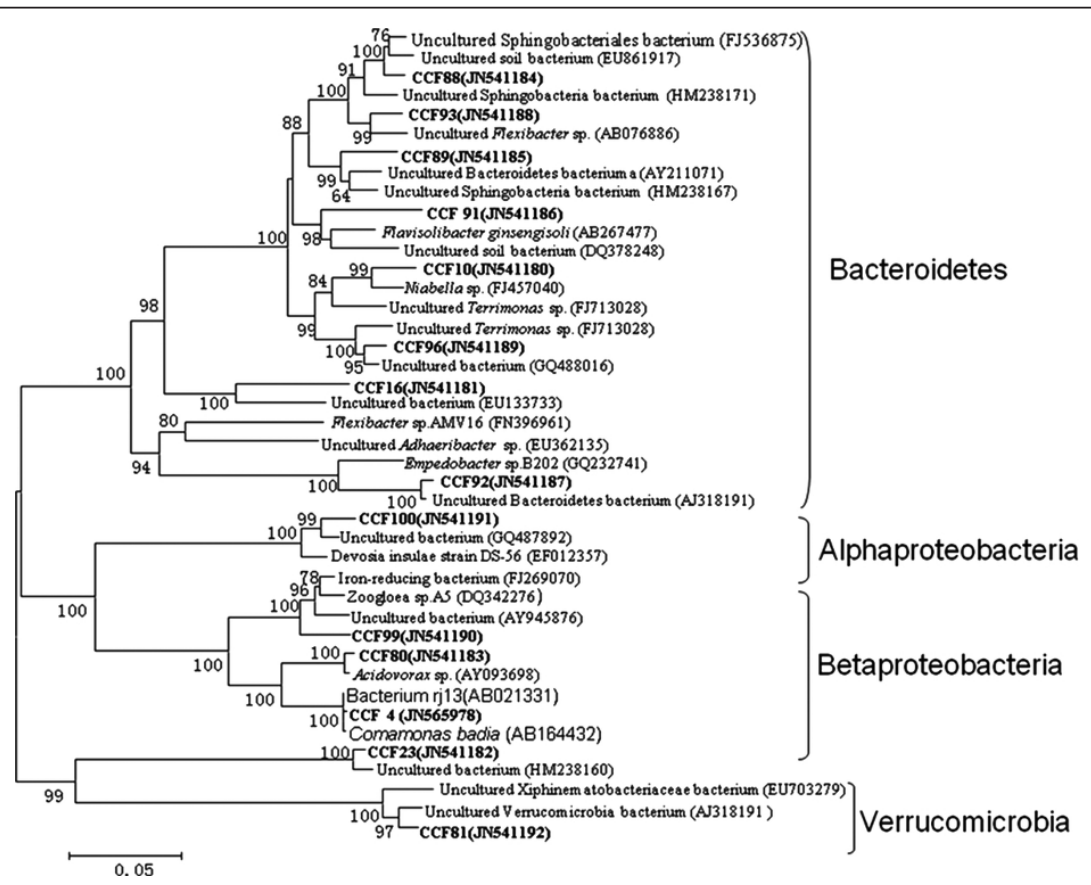

Figure 4 Neighbor-joining tree showing the phylogenetic relationship of bacteria 16S rRNA acquired from carbon felt packed MFC. The clones with labels CCF are from cathodic carbon felt. Bootstrap values are only shown for nodes that had $>50 \%$ support in bootstrap analysis of 1000 replicates in trees. The scale bar indicates 5\% estimated sequence divergence. 


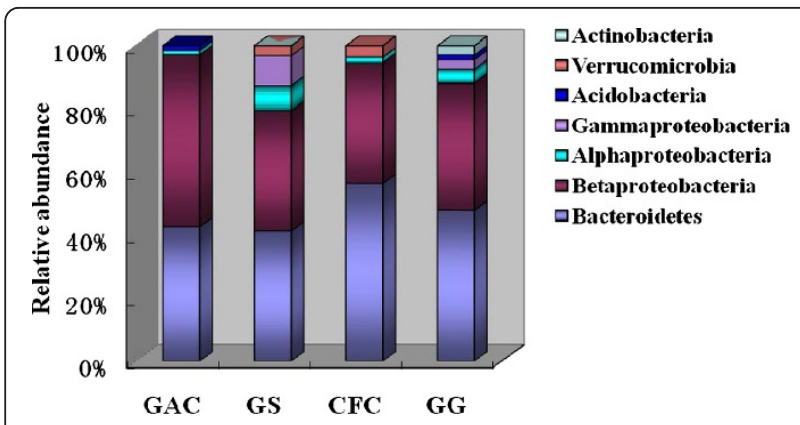

Figure 5 Microbial community distribution and relative abundance in different biocathode materials packed MFCs. GAC (granular activated carbon), CFC (carbon felt cube), GG (granular graphite), GS (granular semicoke).

consisted of $9 \%$ of the library. Pedobacter can only be found in GAC, and the ratio of its population to the library was $3 \%$. The abundance of Comamonas was also high in the GS packed MFC, with a percentage of $21 \%$. Acidovorax and Pigmentiphaga can also be found in GS, and each genus took up approximately $5 \%$ of the microbial community. Sphingomonas, Thermomonas, Burkholderia and Dokdonella can only be retrieved from cathodic communities of the CFC packed MFC, and they covered $6 \%, 5 \%, 3 \%$ and $3 \%$ of the library, respectively. In the case of CFC, the dominate group was similar to GAC and GS, which was also dominated by Comamonas and comprised $30 \%$ of the population. The relative abundance of Flexibacter was high in CFC, and it accounted for 13\% of the library. Different from GAC, GS and CFC packed MFCs, the biofilm on GG was dominated by Acidovorax, which formed $31 \%$ of the community. The microbes belonging to Pigmentiphaga and Flexibacter can also be detected on GG.

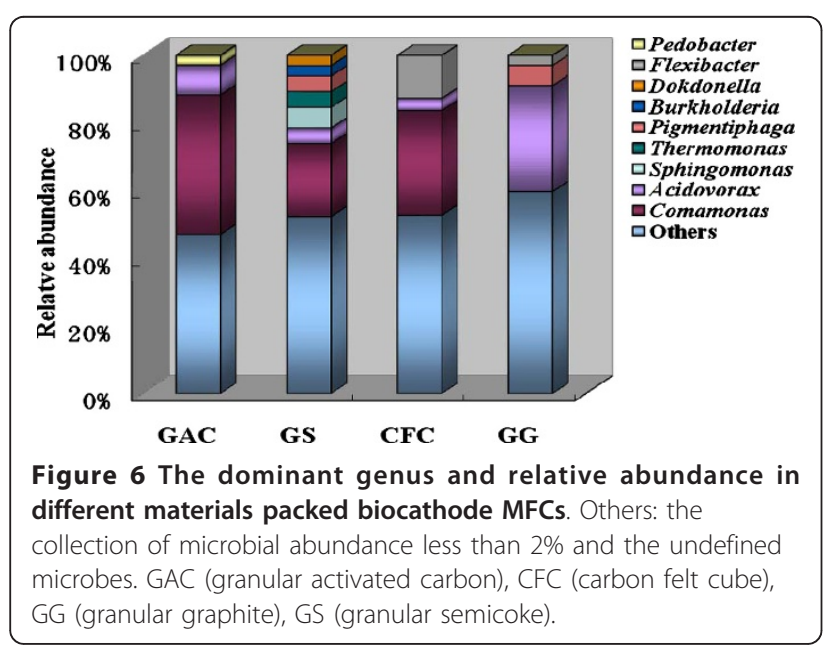

\section{Discussion}

The maximum power densities of GAC, GS,CFC and GG packed MFCs showed a decreasing trend. The specific areas changed in the similar way as the power densities of MFCs with different cathodic materials. It indicated that the power density had positive correlation with specific area, which can be assured that high specific area is profitable for microbial attachment and biological catalytic processes.

Shannon diversity indices results suggested that the diversity was the highest on GS and lowest on GAC. The results showed that biocathode materials had effects on the microbial diversity and evenness. Results on electrical power showed that the power density was the highest in GAC packed MFC, followed by GS, CFC and GG (Additional file 1: Table S1). It indicated that the microbial diversity and evenness in biocathode material was not positively related to the power production. Other studies about anodic microbes also showed that numerical abundance of microorganisms in anodic biofilms was not correlated with the current (Kiely et al. 2010).

Phylogenetic analysis indicated that the biofilm developing on each cathodic surface had some differences. It could be assumed that the microbial members had different affinities for the materials. Bacteroidetes and Betaproteobacteria were predominant in the biofilms sampled from four cathode materials. In a related study, it was also reported that the microbial community composition of an oxygen reducing biocathode was dominated by Bacteroidetes (Rabaey et al. 2008). When nitrate was served as the terminal electron acceptor on the biocathode, Chen et al. (2010) analyzed the microbial community dynamics and the results showed that Betaproteobacteria and Bacteroidetes were the most abundant division of the community. Another study showed that Gammaproteobacteria was the most abundant, followed by uncultured Bacteria and Bacteroidetes (Chen et al. 2008). These results indicated that the microorganisms belonging to proteobacteria and Bacteroidetes play important roles in catalyzing oxygen or nitrate reduction in cathodic compartment.

Electrode materials had an important effect on the type of microbial species in MFCs reactors. Different electrode materials have different microscopic surface structure and conductivity, which in turn affects the adhesion of specific microbes (Rabaey et al. 2004Zhou et al. 2011). In GAC, GS and CFC packed MFCs, Comamonas of Betaproteobacteria was the dominated genus; Acidovorax was the most obvious microbes of GG packed MFC. The powder density was the highest in GAC packed MFC, followed by GS, CFC and GG. Therefore microbes Comamonas and its abundance in cathodic materials might 
have some positive relation to the power generation in GAC, GS and GG packed MFCs, while in GG packed MFC Acidovorax may be correlated with electron transfer. A recent study showed that Comamonas testosteroni displayed a higher power production performance under a high $\mathrm{pH}$ condition in the anode chamber (Juang et al. 2011). The similarities between anode and cathode reducing/oxdizing populations may indicate the capability of many organisms to perform electron transfer both to and from electrodes, such as Shewanella putrefaciens and Geobacter sulfurreducens. Some electrochemically active bacteria in biocathodes have been reported, including Gram-negative and positive bacteria, such as Acinetobacter calcoaceticus, Sphingobacterium multivorum, Micrococcus luteus and Bacillus subtilis, which can catalyze oxygen reduction in biocathode MFCs (Rabaey et al. 2008Cournet et al. 2010). Therefore, the cathodic biofilm in a MFC is composed of diverse populations of bacteria, and they may work together to electron transfer and power production.

In conclusion, electrical results showed that the power density was the highest in GAC packed MFC, followed by GS, CFC and GG. Different biocathode materials had effects on the microbial diversity and evenness, but the differences in microbial diversity and evenness of different biocathode materials were not positively related to the power production. Biocathode materials had an important effect on the type of microbial species in MFCs reactors. The microbes belonging to Bacteroidetes and Proteobacteria were the dominant phyla in the four materials packed biocathode MFCs. Comamonas of Betaproteobacteria might have important effects on electron transfer process of GAC, GS and CFC packed biocathode MFCs, while in GG packed MFC Acidovorax may be correlated with power generation.

\section{Additional material}

Additional file 1: Figure S1. Rarefaction analysis of four clone libraries including GAC (granular activated carbon), CFC (carbon felt cube), GG (granular graphite) and GS (granular semicoke). Table S1. The specific area and power density of different cathodic materials packed MFCs (Wei et al. 2011).

\section{Acknowledgements}

This research was supported by National High Technology Research and Development Program of China (863 Program) (No. 2009AA06Z306) and China Postdoctoral Science Foundation (023208015).

\section{Competing interests}

The authors declare that they have no competing interests.

Received: 23 February 2012 Accepted: 29 March 2012

Published: 29 March 2012

\section{References}

Barberio C, Pagliai L, Cavalieri D, Fani R (2001) Biodiversity and horizontal gene transfer in culturable bacteria isolated from activated sludge enriched in nonylphenol ethoxylates. Res Microbiol 152(1):105-112. doi:10.1016/S09232508(00)01173-6.

Bond DR, Holmes DE, Tender LM, Lovley DR (2002) Electrode-reducing microorganisms that harvest energy from marine sediments. Science 295(5554):483-485. doi:10.1126/science.1066771.

Cárcer DA, Ha PT, Jang JK, Chang IS (2011) Microbial community differences between propionate-fed microbial fuel cell systems under open and closed circuit conditions. Appl Microbiol Biotechnol 89(3):605-612. doi:10.1007/ s00253-010-2903-X.

Carmona-Martinez AA, Harnisch F, Fitzgerald LA, Biffinger JC, Ringeisen BR, Schröder U (2011) Cyclic voltammetric analysis of the electron transfer of Shewanella oneidensis MR-1 and nanofilament and cytochrome knock-out mutants. Bioelectrochem 81(2):74-80. doi:10.1016/j.bioelechem.2011.02.006.

Carbajosa S, Malki M, Caillard R, Lopez MF, Palomares FJ, Martín-Gago JA, Rodríguez N, Amils R, Fernández VM, Lacey ALD (2010) Electrochemical growth of Acidithiobacillus ferrooxidans on a graphite electrode. Biosens Bioelectron 26(2):887-880

Chen GW, Choi SJ, Lee TH, Lee GY, Cha JH, Kim CW (2008) Application of biocathode in microbial fuel cells: cell performance and microbial community. Appl Microbiol Biotechnol 79(3):379-388. doi:10.1007/s00253008-1451-0.

Chen GW, Choi SJ, Lee TH, Lee GY, Cha JH, Kim CW (2010) Microbial community dynamics and electron transfer of a biocathode in microbial fuel cells. Korean J Chem Eng 27(5):1513-1520. doi:10.1007/s11814-010-0231-6.

Cournet A, Délia M-L, Bergel A, Roques C, Bergé M (2010) Electrochemical reduction of oxygen catalyzed by a wide range of bacteria including Gram -positive. Electrochem Commun 12(4):505-508. doi:10.1016/j.elecom.2010.01.026.

Di Cello F, Bevivino A, Chiarini L, Fani R, Paffetti D, Tabacchioni S, Dalmastri C (1997) Biodiversity of a Burkholderia cepacia population isolated from the maize rhizosphere at different plant growth stages. Appl Environ Microbiol 63(11):4485-4493

Gremion F, Chatzinotas A, Harms H (2003) Comparative 165 rDNA and 165 rRNA sequence analysis indicates that Actinobacteria might be a dominant part of the metabolically active bacteria in heavy metalcontaminated bulk and rhizosphere soil. Environ Microbiol 5(10):896-907. doi:10.1046/j.14622920.2003.00484.x

Holmes DE, Nicoll JS, Bond DR, Lovley DR (2004) Potential Role of a Novel Psychrotolerant Member of the Family Geobacteraceae, Geopsychrobacter electrodiphilus gen. nov., sp. nov., in Electricity Production by a Marine Sediment Fuel Cell. Appl Environ Microbiol 70(10):6023-6030. doi:10.1128/ AEM.70.10.6023-6030.2004.

Huang L, Regan JM, Quan X (2011) Electron transfer mechanisms, new applications, and performance of biocathode microbial fuel cells. Bioresour Technol 102(1):316-323. doi:10.1016/j.biortech.2010.06.096.

Jung S, Regan JM (2010) Influence of External Resistance on Electrogenesis, Methanogenesis, and Anode Prokaryotic Communities in Microbial Fuel Cells. Appl Environ Microbiol 77(2):564-571

Juang DF, Yang PC, Chou HY, Chiu $\sqcup$ (2011) Effects of microbial species, organic loading and substrate degradation rate on the power generation capability of microbial fuel cells. Biotechnol Lett 33(11):2147-2160. doi:10.1007/s10529011-0690-9.

Kiely PD, Call DF, Yates MD, Regan JM, Logan BE (2010) Anodic biofilms in microbial fuel cells harbor low numbers of higher-power-producing bacteria than abundant genera. Appl Microbiol Biotechnol 88(1):371-380. doi:10.1007/ s00253-010-2757-2.

Kim JR, Beecroft NJ, Varcoe JR, Dinsdale RM, Guwy AJ, Slade RCT, Thumser A, Avignone-Rossa C, Premier GC (2011) Spatiotemporal development of the bacterial community in a tubular longitudinal microbial fuel cell. Appl Microbiol Biotechnol 90(3):1179-1191. doi:10.1007/s00253-011-3181-y.

Logan BE, Hamelers B, Rozendal R, Schroder U, Keller J, Freguia S, Aelterman P, Verstraete W, Rabaey K (2006) Microbial fuel cells: methodology and technology. Environ SciTechnol 40(17):5181-5192. doi:10.1021/es0605016.

Mao Yp, Zhang LH, Li DM, Shi HF, Liu YD, Cai LK (2010) Power generation from a biocathode microbial fuel cell biocatalyzed by ferro/manganese-oxidizing bacteria. Electrochim Acta 55(27):7804-7808. doi:10.1016/j. electacta.2010.03.004. 
Osman MH, Shah AA, Walsh FC (2010) Recent progress and continuing challenges in bio-fuel cells. Part II: Microbial. Biosens Bioelectron 26(3):953-963. doi:10.1016/j.bios.2010.08.057.

Rabaey K, Boon N, Siciliano SD, Verhaege M, Verstraete W (2004) Biofuel cells select for microbial consortia that self-mediate electron transfer. Appl Environ Microbiol 70(9):5373-5382. doi:10.1128/AEM.70.9.5373-5382.2004.

Rabaey K, Read ST, Clauwaert P, Freguia S, Bond PL, Blackall LL, Keller J (2008) Cathodic oxygen reduction catalyzed by bacteria in microbial fuel cells. ISME J 2(5):519-527. doi:10.1038/ismej.2008.1.

Rabaey K, Rozendal RA (2010) Microbial electrosynthesis-revisiting the electrical route for microbial production. Nat Rev Micro 8(10):706-716. doi:10.1038/ nrmicro2422.

Schloss PD, Handelsman J (2005) Introducing DOTUR, a computer program for defining operational taxonomic units and estimating species richness. Appl Environ Microbiol 71(3):1501-1506. doi:10.1128/AEM.71.3.1501-1506.2005.

Strycharz SM, Glaven RH, Coppi MV, Gannon SM, Perpetua LA, Liu A, Nevin KP, Lovley DR (2011) Gene expression and deletion analysis of mechanisms for electron transfer from electrodes to Geobacter sulfurreducens. Bioelectrochem 80(2):142-150. doi:10.1016/j.bioelechem.2010.07.005.

Tamura K, Dudley J, Nei M, Kumar S (2007) MEGA4: Molecular evolutionary genetics analysis (MEGA) software version 4.0. Mol Bio Evol 24(8):1596-1599. doi:10.1093/molbev/msm092.

Wei JC, Liang P, Cao XX, Huang X (2011) Use of inexpensive semicoke and activated carbon as biocathode in microbial fuel cells. Bioresour Technol 102(22):10431-10435. doi:10.1016/j.biortech.2011.08.088.

Xia X, Cao XX, Liang P, Huang X, Yang SP, Zhao GG (2010) Electricity generation from glucose by a Klebsiella sp. in microbial fuel cells. Appl Microbiol Biotechnol 87(1):383-390. doi:10.1007/s00253-010-2604-5.

Zhang Y, Min B, Lp H, Angelidaki I (2011) Electricity generation and microbial community response to substrate changes in microbial fuel cell. Bioresour Technol 102(2):1166-1173. doi:10.1016/.jbiortech.2010.09.044.

Zhou M, Chi M, Luo J, He H, Jin T (2011) An overview of electrode materials in microbial fuel cells. J Power Sources 196(10):4427-4435. doi:10.1016/j. jpowsour.2011.01.012.

doi:10.1186/2191-0855-2-21

Cite this article as: Sun et al:: Microbial community analysis in

biocathode microbial fuel cells packed with different materials. $A M B$ Express 2012 2:21.

\section{Submit your manuscript to a SpringerOpen ${ }^{\mathcal{O}}$ journal and benefit from:}

- Convenient online submission

- Rigorous peer review

- Immediate publication on acceptance

- Open access: articles freely available online

- High visibility within the field

- Retaining the copyright to your article

Submit your next manuscript at $\gg$ springeropen.com 